\title{
Protein typing of circulating microvesicles allows real-time monitoring of glioblastoma therapy
}

\section{Citation}

Shao, Huilin, Jaehoon Chung, Leonora Balaj, Alain Charest, Darell D Bigner, Bob S Carter, Fred H Hochberg, Xandra 0 Breakefield, Ralph Weissleder, and Hakho Lee. 2012. "Protein Typing of Circulating Microvesicles Allows Real-Time Monitoring of Glioblastoma Therapy." Nature Medicine 18 (12): 1835-40. https://doi.org/10.1038/nm.2994.

\section{Permanent link}

http://nrs.harvard.edu/urn-3:HUL.InstRepos:41384266

\section{Terms of Use}

This article was downloaded from Harvard University's DASH repository, and is made available under the terms and conditions applicable to Other Posted Material, as set forth at http:// nrs.harvard.edu/urn-3:HUL.InstRepos:dash.current.terms-of-use\#LAA

\section{Share Your Story}

The Harvard community has made this article openly available. Please share how this access benefits you. Submit a story. 


\title{
Protein typing of circulating microvesicles allows real-time monitoring of glioblastoma therapy
}

\author{
Huilin Shao ${ }^{1,2}$, Jaehoon Chung ${ }^{1}$, Leonora Balaj ${ }^{3}$, Alain Charest ${ }^{4}$, Darell D. Bigner ${ }^{5}$, Bob S.

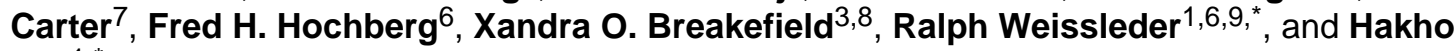 \\ Lee $^{1,{ }^{*}}$ \\ ${ }^{1}$ Center for Systems Biology, Massachusetts General Hospital, 185 Cambridge St, Boston, MA \\ 02114 \\ ${ }^{2}$ Harvard Biophysics Program, Harvard Medical School, Boston, MA 02115 \\ ${ }^{3}$ Neuroscience Center, Department of Neurology, Massachusetts General Hospital, Charlestown \\ Navy Yard, Boston, MA 02129 \\ ${ }^{4}$ Molecular Oncology Research Institute, Tufts University School of Medicine, Boston, MA 02111 \\ ${ }^{5}$ Brain Tumor Center, Department of Pathology, Duke University Medical Center, Durham, NC \\ 27710 \\ ${ }^{6}$ Massachusetts General Hospital Cancer Center, Boston, MA 02114 \\ ${ }^{7}$ Division of Neurological Surgery, UCSD School of Medicine, San Diego, CA 92103 \\ ${ }^{8}$ Program in Neuroscience, Harvard Medical School, Boston MA 02114 \\ ${ }^{9}$ Department of Systems Biology, Harvard Medical School, Boston, MA 02115
}

\section{Abstract}

Glioblastomas shed large quantities of small, membrane-bound microvesicles (MVs) into the circulation. While these hold promise as potential biomarkers of therapeutic response, their identification and quantitation remain challenging. Here, we describe a highly sensitive and rapid analytical technique for profiling circulating MVs directly from blood samples of glioblastoma patients. MVs, introduced onto a dedicated microfluidic chip, are labeled with target-specific magnetic nanoparticles and detected by a miniaturized nuclear magnetic resonance system. Compared with current methods, this integrated system has a much higher detection sensitivity, and can differentiate glioblastoma multiforme (GBM) MVs from non-tumor host cell-derived MVs. We also show that circulating GBM MVs can serve as a surrogate for primary tumor mutations and a predictive metric of treatment-induced changes. This platform could provide both an earlier indicator of drug efficacy and a potential molecular stratifier for human clinical trials.

Many cancers shed materials into the peripheral circulation. These appear as circulating tumor cells (CTCs) ${ }^{1}$ and soluble proteins $s^{2,3}$, and are being exploited as surrogate markers of tumor staging and response to therapy. Systemic and intracranial tumors also release

\footnotetext{
*Corresponding authors and equal contribution: H. Lee, PhD Center for Systems Biology Massachusetts General Hospital 185 Cambridge St, CPZN 5206 Boston, MA, 02114 617-726-8226 hlee@mgh.harvard.edu R. Weissleder, MD, PhD Center for Systems Biology Massachusetts General Hospital 185 Cambridge St, CPZN 5206 Boston, MA, 02114 617-726-8226 rweissleder@mgh.harvard.edu.

Author Contributions H.S., R.W. and H.L. designed the study. H.S., J.C., L.B. and H.L. performed the experiments. H.S., J.C., R.W and H.L. analyzed the data and wrote the manuscript. A.C. generated the mouse T103 model. D.D.B. recommended GBM biomarkers and generated the EGFRvIII antibody. F.H.H. and X.O.B. coordinated the clinical study and analyzed the results.
} 
microvesicles (MVs) $)^{4-6}$ into the peripheral circulation. In particular, tumors of the central nervous system, lying behind a partially intact blood brain barrier, often do not release CTCs nor are they commonly associated with detectable soluble protein biomarkers. Large quantities of MVs, however, have been found within blood of patients with glioblastoma multiforme $(\mathrm{GBM})^{3,5}$, and these thus offer new hope for treatment monitoring of this devastating disease.

MVs in circulation are made up of membrane-bound vesicles (50 $\mathrm{nm}-1 \mu \mathrm{m}$ in diameter), which differ in their cellular origin, abundance and biogenesis ${ }^{4}$. The population includes exosomes (50 - $100 \mathrm{~nm}$ and positive for CD63, HSP90, Flotillins) released from multivesicular endosomes, larger shed microvesicles, membrane particles, apoptotic vesicles and exosome-like vesicles originating from multivesicular bodies of other cell organelles ${ }^{4,7,8}$. Different subtypes of MVs can have overlapping size and often co-purify if separated by size only ${ }^{4,9}$. MVs contain cell surface proteins ${ }^{10}$, including EGFR and EGFRvIII $^{6,11}$, as well as RNA ${ }^{5}$ and $\mathrm{DNA}^{12}$. Current analyses generally require large numbers of MVs to be concentrated and processed using time-consuming Western blotting or enzyme-linked immunosorbent assays (ELISA), making them impractical in a typical clinical setting.

Herein, we describe a highly sensitive and rapid analytical technique for profiling proteins in MVs from GBM cell cultures and from GBM patient blood samples (circulating MVs). We use both size and immunoaffinity (vesicles $50-150 \mathrm{~nm}$ and CD63-positive) to define a population of circulating MVs, which consist primarily of exosomes. MVs are labeled with target-specific magnetic nanoparticles (MNPs), and detected by a miniaturized (micro) nuclear magnetic resonance $(\mu \mathrm{NMR})$ system $^{13,14}$. A prototype $\mu$ NMR system was previously used to detect whole tumor cells $(>10 \mu \mathrm{m} \text { target size range })^{15}$. Adapting $\mu$ NMR to MV detection, however, presented significant engineering challenges since these targets are smaller than tumor cells by $1-2$ orders of magnitude. We thus developed a new microfluidic system and analytical technology specifically for MV detection and profiling in GBM patients, that can differentiate glioma-derived MVs from host cell-derived MVs. Employing this technology, we describe findings to evaluate the comparative protein profiles of glioma-derived MVs against those from parental GBM cells. We likewise report on the ability of this system to detect specific circulating MVs from blood of GBM patients and non-GBM control subjects, and whether circulating MVs can be used in longitudinal studies to monitor and predict response to GBM therapies.

\section{RESULTS}

\section{Magnetic nanosensor technology for MV detection}

GBM cell lines in culture produced abundant MVs (Fig. 1a). MV counting based on nanoparticle tracking analysis (NTA; Supplementary Fig. 1a) reported a typical concentration of $10^{8}-10^{9} \mathrm{MVs} \mathrm{mL}^{-1}$ in culture media. In situ scanning electron microscopy analysis of MVs on cell surface revealed that many of the MVs were saucershaped $^{16}$, a pattern typical of exosomes (Fig. 1b). For detection by the microfluidic $\mu$ NMR, we purified and labeled MVs with magnetic nanoparticles (MNPs, core diameter $7 \mathrm{~nm}$ ) by targeting MV protein markers (Fig. 1c). Such magnetic labeling renders MV superparamagnetic, which results in faster decay of the ${ }^{1} \mathrm{H}$ NMR signal. The decay rate $\left(R_{2}\right)$ is proportional to the MNP concentration, thus enabling the quantitation of target MV protein concentration.

We used a two-step bioorthogonal approach (BOND-2; Fig. 1d) for MNP labeling. MV protein markers were first targeted with antibodies modified with trans-cyclooctene (TCO), and then coupled with MNPs derivatized with 1,2,4,5-tetrazine (TZ). The fast, covalent 
cycloaddition between TZ and TCO maximizes MNP binding and increases the magnetic signals $\left(R_{2}\right)$ by $>300 \%$ compared to that achieved by direct MNP-antibody conjugation ${ }^{17}$. Because both TZ and TCO are small ( 200 Daltons each), this chemical conjugation does not appreciably increase the size of the antibody or the MNP. It is thus possible to use sizeselective filtration to remove excess antibodies or MNPs, while retaining targeted MVs. Figure 1e shows a prototype device developed for clinical, point-of-care MV analysis. It contains three essential components: i) a chaotic mixer for reacting MVs with antibodies and MNPs, ii) a membrane filter for washing and concentrating targeted MVs, and iii) a microcoil for NMR detection (sample volume: $1 \mu \mathrm{L}$ ). MVs purified by the microfluidic device typically have a size distribution ranging $50-150 \mathrm{~nm}$ (with > 75\% smaller than 120 $\mathrm{nm}$ in diameter), due to the cut-off sizes of membrane filtration (Supplementary Fig. 1b). To streamline the assay procedure (Supplementary Fig. 2), the fluidic flow is controlled by automated pneumatic valves (Supplementary Fig. 3).

\section{Optimized assay for MV protein typing}

We hypothesized that in multicellular environments, CD63 expression could be used as an internal measure of total MV counts from different cell sources (Supplementary Fig. 4a) ${ }^{4,7}$. Figure 2 summarizes the results of the validation study. When MVs were analyzed for CD63 expression, the corresponding $R_{2}$ changes $\left(\Delta R_{2} \mathrm{CD} 63\right)$ were found to be linearly proportional to MV counts, which were independently confirmed by NTA (Fig. 2a). Importantly, these $R_{2}$ changes were statistically identical even among MVs from different cell lines $(P>0.16)$. The $\mu$ NMR measurements were highly reproducible and accurate with $<1 \%$ instrumental errors. The expression levels of other protein biomarkers were then determined by normalizing the marker-associated $\Delta R_{2}{ }^{\mathrm{mAb}}$ by $\Delta R_{2}{ }^{\mathrm{CD} 63}\left(\xi_{\mathrm{mAb}}=\Delta R_{2}{ }^{\mathrm{mAb}} / \Delta R_{2}{ }^{\mathrm{CD} 63}\right)$. Such normalization automatically accounts for differences in MV counts, obviating the need to quantify MVs by other measures such as NTA. The $\mu$ NMR also showed excellent agreement $\left(R^{2}>99 \%\right)$ with fluorescence ELISA (Fig. 2b), and its detection sensitivity (Fig. $2 \mathrm{c}$ ) far surpassed that of other analytical methods, being $10^{4}, 10^{3}$, and $10^{2}$-fold more sensitive than Western blotting, ELISA, and NTA respectively (Fig. 2d and Supplementary Fig. $4 b$ ). Since $\mu$ NMR detection sensitivity depends on biomarker protein integrity, we also tested the detection level as a function of time (Supplementary Fig. 5a) and showed that signal degradation can be largely prevented by fixation (Supplementary Fig. 5b).

\section{MV molecular signature for GBM detection}

We next determined whether GBM-derived MVs show similar protein profiles to that of intact parental cells, and whether molecular markers could be used to differentiate between GBM and host cell (non-GBM) MVs. Based on prior reports ${ }^{3,4,18}$, we measured the following seven extravesicular and two intravesicular protein markers: epidermal growth factor receptor $(\mathrm{EGFR})^{11}$, platelet-derived growth factor receptor (PDGFRa) ${ }^{19}$, podoplanin $(\mathrm{PDPN})^{20}$, ephrin type-A receptor $2(\mathrm{EphA} 2)^{21}$ for their elevated expressions in glioma; EGFRvIII $^{6}$ and cytosolic isocitrate dehydrogenase 1 mutation (IDH1 R132H) ${ }^{22,23}$ for their specific expressions in glioma; cytosolic heat shock protein 90 (HSP90) ${ }^{3,4}$ as a positive control for MVs; CD41 ${ }^{24}$ and major histocompatibility complex class II (MHCII) ${ }^{4}$ to represent host cell markers. Comparative analyses confirmed that MVs indeed reflect the protein profiles of their parental cells (Fig. 3a). GBM-derived MVs exhibit a distinct molecular signature; elevated expressions of EGFR, EGFRvIII, PDPN and IDH1 R132H together allowed effective discrimination of GBM and host cell MVs.

We next profiled MVs from blood samples of GBM patients $(n=24)$ and healthy volunteers $(n=8$; Supplementary Table 1). Samples for this study had been selected in a blinded fashion but were enriched in samples positive for EGFR amplification or EGFRvIII mutation. For each patient, there was considerable heterogeneity in the expression levels of 
individual markers (Supplementary Fig. 6a). The waterfall plot for each marker revealed a broad spectrum of expression level (Fig. 3b). Consistent with prior report ${ }^{22}$, IDH1 R132H, albeit highly specific to GBM, showed lower prevalence in primary GBM patients. The accuracy of each marker in GBM detection, obtained from the receiver operating characteristic (ROC) curves, was $<76 \%$ (Fig. 3c and Supplementary Fig. 6b). However, by combining the results of all four markers, the accuracy increased to $>90 \%$ (Supplementary Table 2).

\section{Efficacy of drug treatment revealed by MVs}

We investigated the potential use of MVs for monitoring treatment responses. Two drug regimens were selected: 1) an alkylating agent, temozolomide (TMZ), which is a first line adjuvant drug currently used with concomitant radiation therapy for the treatment of newly diagnosed GBM; and 2) a HSP90 inhibitor, geldanamycin (17AAG), whose binding to HSP90 can enhance the degradation of signaling molecules (e.g., EGFR) ${ }^{25-27}$. Treatment effects on T103 mouse GBM cells and MVs are compared in Supplementary Fig. 7 (for TMZ) and Fig. 4 (for geldanamycin). TMZ treatment did not elicit significant changes in the cellular expression of CD63, EGFR or EGFRvIII, as determined by flow cytometry and Western blotting (Supplementary Fig. 7a). Likewise, corresponding $\mu$ NMR assays on MVs reported comparable expression levels for EGFR and EGFRvIII (Supplementary Fig. 7b). TMZ treatment, however, resulted in a dose-dependent decrease in both cell and MV counts (Supplementary Fig. 7c). Total MV expression of CD63, EGFR, and EGFRvIII, as measured by the $\mu \mathrm{NMR}$, thus showed a similar dose-dependent decline, with increasing concentration of TMZ applied (Supplementary Fig. 7d).

In contrast to TMZ, geldanamycin resulted in a significant decrease in EGFR and EGFRvIII expression, but not in CD63, for both cells and MVs (Figs. 4a and b). Consequently, as MV numbers decreased in proportion to drug concentration (a change attributed to cell loss; Fig. 4c), the total MV expression of EGFR and EGFRvIII each showed a much more pronounced decline than MV-CD63 (Fig. 4d). To account for such additive effects, we defined a drug response index $(\mathrm{RI})$ as $\mathrm{RI}=\left[(1-\Delta n)+\Sigma\left(1-\Delta \xi_{k}\right)\right] / N$, where $1-\Delta n$ and $1-\Delta \xi_{k}$ are the relative changes in glioma MV numbers and MV biomarker expression levels $\left(\xi_{k}\right)$, respectively, and $N$ is the total number of markers monitored. Conversely, the complementary of RI was defined as a tumor progression index $(\mathrm{TPI}=1-\mathrm{RI})$. A plot of RI captured the drug efficacies of TMZ and geldanamycin (Fig. 4e). These findings were corroborated in repeated studies with the human GBM GLI36vIII line, which showed nearly identical trends (Fig. 4f and Supplementary Fig. 8), indicating that normalized detection of MV biomarkers is highly sensitive to reveal the differences between treatment mechanisms.

\section{Circulating MVs predict drug efficacy in vivo}

We applied the platform to monitor treatment effects in vivo. We first used a mouse model with xenografted human $\mathrm{GBM}^{28}$. Circulating MVs in cohorts of T103 tumor-bearing mice were profiled during tumor growth as well as following TMZ treatment $(80 \mathrm{mg} / \mathrm{kg}$ daily). In untreated animals, the tumor progression index (TPI) increased over time and paralleled tumor volume measured by MRI (Fig. 5a). In TMZ treated animals, TPI changes occurred several days prior to reductions in tumor volume as measured by imaging (Fig. 5b). To visualize the temporal onset of therapeutic effects, we plotted an efficacy index $\left(\eta_{M V}\right)$, defined as the temporal change in 1/TPI. As shown in Fig. 5c, the efficacy index $\eta_{M V}$ was close to zero for expanding tumors. Upon TMZ administration, however, $\eta_{M V}$ rose rapidly, indicating the effectiveness of the treatment.

We next extended the study to clinical GBM patients whose blood samples were collected prior to and after standard-of-care TMZ/radiation treatment (Supplementary Table 3). In this 
longitudinal study, the tumor progression index (TPI) was evaluated upon samples became available. Responder and non-responder status was later defined by a neuro-oncologist without knowledge of the $\mu$ NMR results, and based on subsequent clinical and MRI data. TPI allowed the identification and prediction of treatment outcomes, especially for nonresponding cases (Fig. 5d). The corresponding efficacy index $\left(\eta_{M V}\right)$ also showed significant difference $(P<0.005)$ between responders and non-responders (Fig. 5e).

\section{DISCUSSION}

GBM is the most common primary malignancy of the central nervous system ${ }^{29,30}$.

Amplification of EGFR is the most frequent genetic abnormality associated with GBM, and EGFR overexpression has been shown in up to $85 \%$ of cases ${ }^{29}$. GBM also often expresses EGFRvIII, a genomic deletion variant of EGFR that is constitutively active and highly oncogenic ${ }^{31,32}$. It is likely that the recent identification of circulating MVs containing EGFRvIII specific RNA ${ }^{5}$ and GBM associated proteins ${ }^{10}$ will not only be immediately relevant to this subset of GBM patients, but could also be expanded to other GBM-mutation evaluation. Likewise, circulating MVs may provide new avenues for cancer diagnostics and expand our understanding of cellular communication.

Evaluating circulating MVs could lead to a paradigm shift in clinical care. Phase 1 and phase 2 trials of targeted agents presently require molecular stratification of GBM tumors. In addition, there remains an urgent need to provide sequential indices of tumor molecular response to these agents. While imaging remains useful as a clinical tool, the standard RECIST (Response Evaluation Criteria In Solid Tumors) and volumetric criteria of response are insensitive therapeutic markers in patients receiving vascular-targeted agents such as Avastin. New and more sensitive imaging approaches ${ }^{33-36}$ are currently in development; however, they are not universally available, are often expensive ${ }^{37}$ and impractical for rapid sequential evaluations. As a result, there has been intense interest in finding serologic biomarkers for GBM.

Our findings show that GBM-derived circulating MVs can be rapidly detected in clinical blood samples with high sensitivity using a nanotechnology-inspired biosensor. The system combines on-chip micro-filtration and $\mu$ NMR principles, to enable quantitative detection of MV numbers and protein expression. Measurements are performed on small sample volumes without the need for extensive purification or time-consuming detection techniques. By employing a bioorthogonal targeting approach to specifically target and densely pack MNPs onto MVs, the current platform has achieved a detection sensitivity that surpasses standard ELISA and flow cytometry analyses by several orders of magnitude (Fig. 2). We believe that this could be further enhanced with the use of newer magnetic nanomaterials (with higher magnetization) ${ }^{38}$, improved assay types, additional amplification steps, and new bioorthogonal approaches. Likewise, further device optimization are expected. By incorporating differential, multistep filtering system, MVs can be isolated from whole cells; multiple microcoils can be embedded for extensive parallel profiling of a larger number of MV proteins. Such system could realize a comprehensive yet portable lab-on-a-chip for MV analysis.

We further envision other clinical applications in which protein typing of circulating MVs would be useful. The above-described methodology could be extended to examine other primary tumors, particularly since many cancers secrete much greater quantities of circulating MVs than CTCs. It could also be adapted to monitor circulating MVs in a variety of inflammatory and infectious diseases, using blood samples, cerebral spinal fluid, urine, saliva or other biofluids. With its capacity for molecular diagnostics at the bedside, the developed platform could potentially redefine the current standard-of-care for patients. 


\section{Materials and Methods}

\section{Cell culture}

The following human GBM cell lines were cultured in Dulbecco's modified essential medium (DMEM, Cellgro) containing 10\% fetal bovine serum (FBS, Cellgro) and supplemented with penicillin-streptomycin (Cellgro): SkMG3 was provided by Dr. Timothy Chan, Memorial Sloan-Kettering Cancer Center; GBM20/3, GLI36vIII (overexpressing human EGFRvIII), and GLI36R132H (overexpressing human IDH1 R132H mutant protein) were provided by Dr. Xandra Breakefield, Massachusetts General Hospital (MGH); LNZ308 was provided by Dr. Mikael Pittet, MGH; A172 was purchased from American Type Culture Collection. Mouse model cell lines overexpressing human wild-type EGFR and human EGFRvIII (T103: WT/vIII, T042: vIII only) were generated as previously reported, and provided by Dr. Alain Charest (Tufts University). These cell lines were cultured on gelatin and in DMEM containing 20\% FBS, supplemented with penicillinstreptomycin. Normal human brain microvascular endothelial cells (HBMVECs, Cell Systems) were cultured in endothelial basal medium supplemented with SingleQuots (Lonza). Normal human astrocytes (NHA, Lonza) were cultured in astrocyte basal medium supplemented with SingleQuots, as recommended by the manufacturer.

\section{Microvesicle (MV) isolation from cell culture and quantification}

Cells at passage 1-15 were cultured in MV-free medium (DMEM containing 5\% depleted FBS). After 48 hours, conditioned medium from $\sim 10^{7}$ cells was collected and filtered through a $0.2 \mu \mathrm{m}$ filter (Millipore), and MVs were purified by differential centrifugation as described previously ${ }^{5,12}$. MV numbers were determined by Nanosight LM10 nanoparticle characterization system (Nanosight). All nanoparticle tracking analyses (NTA) were done with identical experiment settings. For optimal results, MV concentrations were adjusted to obtain $\sim 50 \mathrm{MVs}$ in the field of view.

\section{MV isolation from clinical samples}

Blood samples were collected from healthy donors and GBM patients under protocols approved by the MGH Institutional Review Board. Blood was collected in vacutainer tubes (Becton Dickinson). Buffy coat was obtained via centrifugation and remaining plasma was then sterile-filtered through a $0.8 \mu \mathrm{m}$ filter (Millipore). All clinical samples were frozen at $-80{ }^{\circ} \mathrm{C}$ within two hours of collection. Thawed samples were processed for MV isolation using the same method described above. Clinical samples were processed unidentified for $\mu$ NMR detection.

\section{MV labeling and detection}

Isolated MVs were resuspended in PBS and labeled with antibodies $(10 \mu \mathrm{g} / \mathrm{mL})$ for 45 minutes at $4{ }^{\circ} \mathrm{C}$. The samples were then washed and membrane-filtered (Nuclepore, Whatman) to concentrate the MVs and to remove unbound antibodies. For ELISA measurements, fluorescein (FITC)-conjugated antibodies were used for targeting and scanning with fluorescence reader (Safire, Tecan). For $\mu$ NMR detection with the microfluidic chip, MVs were mixed with TCO-modified antibodies. The antibody-targeted MVs and CLIO-TZ were then mixed within the microfluidic device and processed for NMR measurements (see details in Supplementary Fig. 2). For detection of intravesicular proteins, MV lysates were immuno-captured by antibody-conjugated beads ( $500 \mathrm{~nm}$ in diameter), incubated with TCO-modified detection antibody and processed for NMR measurements after coupling with CLIO-TZ in the microfluidic device. All experiments were performed with TCO-modified isotype control antibodies to determine nonspecific background 
binding ${ }^{17}$. MV biomarker expression profiles were normalized by their CD63 expression to account for variations in MV numbers.

\section{$\mu$ NMR measurement}

We performed NMR measurements using a previously described miniaturized NMR relaxometer ${ }^{14}$. The operating magnetic field, generated by a portable permanent magnet, was $0.5 \mathrm{~T}$. The $R_{2}$ relaxation was measured on $1 \mu \mathrm{L}$ sample volumes using Carr-PurcellMeiboom-Gill pulse sequences with the following parameters: echo time, $4 \mathrm{~ms}$; repetition time, $1 \mathrm{~s}$; the number of $180^{\circ}$ pulses per scan, 50; the number of scans, 8 . All measurements were done in triplicate and data is displayed as mean \pm standard error of mean.

\section{Clinical samples}

The study was approved by the MGH Institutional Review Board, and informed consent was obtained from all participants. For the profiling study, we obtained clinical blood samples prior to tumor excision from patients, assumed to harbor newly diagnosed or recurrent glioblastoma. The diagnosis was subsequently confirmed by neuropathologic examination. We subdivided glioblastoma into glioblastoma (GBM) and glioblastoma with oligodendroglial component (GBMO) according to the WHO classification of tumors of the nervous system. EGFR amplification was determined on pathologic tissues using standardized approaches. EGFRvIII mutations were evaluated on tumor tissues using techniques previously described ${ }^{5}$. Peripheral white blood cell (WBC) count was determined at the time of initial operation. MRI was performed prior to operation and evaluated for the presence of gadolinium enhancement (MRI+/-) and the maximum cross-sectional size of the tumor $\left(\right.$ area in $\mathrm{cm}^{2}$ ). For the longitudinal treatment response evaluation, blood samples were collected from the same patients before and after treatment. Responder and non-responder status was defined by a neuro-oncologist based on subsequent clinical and MRI data.

\section{Supplementary Material}

Refer to Web version on PubMed Central for supplementary material.

\section{Acknowledgments}

The authors thank Dr. T. Reiner for preparing TCO, Dr. N. Sergeyev for synthesizing MNPs, Dr. S. Hilderbrand for synthesizing reactive TZ, Dr. M. Pittet for LNZ308 cells, Dr. T. Chan for SkMG3 cells, and Drs. M. Liong and A. Ghazani for assay assistance, B. Marinelli for $\mu$ NMR measurements, C. Min for software implementation, M. McKee for transmission electron microscopy, Dr. J. Skog for advice on NTA measurements, L. Zhu and S. Sivaraman for technical assistance and Dr. Y. Fisher-Jeffes for critically reading the manuscript. Special thanks to Drs. Carter, Castro, Carlson and clinical colleagues for many helpful discussions. This work was supported in part by: U54CA151884, R01EB010011, R01EB004626, P01CA069246, P50CA86355, U01CA141556, U24CA092782, R21CA14122; H. Shao received a B.S.-Ph.D. National Science Scholarship awarded by the Singapore Agency for Science, Technology and Research; A. Charest received an American Cancer Society Research Scholar Award 117409 .

\section{References}

1. Maheswaran S, et al. Detection of mutations in EGFR in circulating lung-cancer cells. N Engl J Med. 2008; 359:366-377. [PubMed: 18596266]

2. Kulasingam V, Pavlou MP, Diamandis EP. Integrating high-throughput technologies in the quest for effective biomarkers for ovarian cancer. Nat Rev Cancer. 2010; 10:371-378. [PubMed: 20383179]

3. Simpson RJ, Lim JW, Moritz RL, Mathivanan S. Exosomes: proteomic insights and diagnostic potential. Expert Rev Proteomics. 2009; 6:267-283. [PubMed: 19489699]

4. Thery C, Ostrowski M, Segura E. Membrane vesicles as conveyors of immune responses. Nat Rev Immunol. 2009; 9:581-593. [PubMed: 19498381] 
5. Skog J, et al. Glioblastoma microvesicles transport RNA and proteins that promote tumour growth and provide diagnostic biomarkers. Nat Cell Biol. 2008; 10:1470-1476. [PubMed: 19011622]

6. Al-Nedawi K, et al. Intercellular transfer of the oncogenic receptor EGFRvIII by microvesicles derived from tumour cells. Nat Cell Biol. 2008; 10:619-624. [PubMed: 18425114]

7. Simons M, Raposo G. Exosomes--vesicular carriers for intercellular communication. Curr Opin Cell Biol. 2009; 21:575-581. [PubMed: 19442504]

8. Lee TH, et al. Microvesicles as mediators of intercellular communication in cancer--the emerging science of cellular `debris'. Semin Immunopathol. 2011; 33:455-467. [PubMed: 21318413]

9. Cocucci E, Racchetti G, Meldolesi J. Shedding microvesicles: artefacts no more. Trends Cell Biol. 2009; 19:43-51. [PubMed: 19144520]

10. Graner MW, et al. Proteomic and immunologic analyses of brain tumor exosomes. FASEB J. 2009; 23:1541-1557. [PubMed: 19109410]

11. Sanderson MP, et al. Generation of novel, secreted epidermal growth factor receptor (EGFR/ ErbB1) isoforms via metalloprotease-dependent ectodomain shedding and exosome secretion. $\mathrm{J}$ Cell Biochem. 2008; 103:1783-1797. [PubMed: 17910038]

12. Balaj L, et al. Tumour microvesicles contain retrotransposon elements and amplified oncogene sequences. Nat Commun. 2011; 2:180. [PubMed: 21285958]

13. Lee H, Sun E, Ham D, Weissleder R. Chip-NMR biosensor for detection and molecular analysis of cells. Nat Med. 2008; 14:869-874. [PubMed: 18607350]

14. Issadore D, et al. Miniature magnetic resonance system for point-of-care diagnostics. Lab Chip. 2011; 11:2282-2287. [PubMed: 21547317]

15. Haun JB, et al. Micro-NMR for rapid molecular analysis of human tumor samples. Sci Transl Med. 2011; 3:71ra16.

16. Thery C, Zitvogel L, Amigorena S. Exosomes: composition, biogenesis and function. Nat Rev Immunol. 2002; 2:569-579. [PubMed: 12154376]

17. Haun JB, Devaraj NK, Hilderbrand SA, Lee H, Weissleder R. Bioorthogonal chemistry amplifies nanoparticle binding and enhances the sensitivity of cell detection. Nat Nanotechnol. 2010; 5:660665. [PubMed: 20676091]

18. Thery C, Amigorena S, Raposo G, Clayton A. Isolation and characterization of exosomes from cell culture supernatants and biological fluids. Curr Protoc Cell Biol. 2006; Chapter 3(Unit 3.22)

19. Fleming TP, et al. Amplification and/or overexpression of platelet-derived growth factor receptors and epidermal growth factor receptor in human glial tumors. Cancer Res. 1992; 52:4550-4553. [PubMed: 1322795]

20. Mishima K, et al. Increased expression of podoplanin in malignant astrocytic tumors as a novel molecular marker of malignant progression. Acta Neuropathol. 2006; 111:483-488. [PubMed: 16596424]

21. Wykosky J, Gibo DM, Stanton C, Debinski W. EphA2 as a novel molecular marker and target in glioblastoma multiforme. Mol Cancer Res. 2005; 3:541-551. [PubMed: 16254188]

22. Parsons DW, et al. An integrated genomic analysis of human glioblastoma multiforme. Science. 2008; 321:1807-1812. [PubMed: 18772396]

23. Dang L, et al. Cancer-associated IDH1 mutations produce 2-hydroxyglutarate. Nature. 2009; 462:739-744. [PubMed: 19935646]

24. Italiano JEJ, Mairuhu AT, Flaumenhaft R. Clinical relevance of microparticles from platelets and megakaryocytes. Curr Opin Hematol. 2010; 17:578-584. [PubMed: 20739880]

25. Pedersen NM, et al. Expression of epidermal growth factor receptor or ErbB3 facilitates geldanamycin-induced down-regulation of ErbB2. Mol Cancer Res. 2009; 7:275-284. [PubMed: 19208749]

26. Trepel J, Mollapour M, Giaccone G, Neckers L. Targeting the dynamic HSP90 complex in cancer. Nat Rev Cancer. 2010; 10:537-549. [PubMed: 20651736]

27. Zhu H, et al. The novel Hsp90 inhibitor NXD30001 induces tumor regression in a genetically engineered mouse model of glioblastoma multiforme. Mol Cancer Ther. 2010; 9:2618-2626. [PubMed: 20643786] 
28. Zhu H, et al. Oncogenic EGFR signaling cooperates with loss of tumor suppressor gene functions in gliomagenesis. Proc Natl Acad Sci U S A. 2009; 106:2712-2716. [PubMed: 19196966]

29. Comprehensive genomic characterization defines human glioblastoma genes and core pathways. Nature. 2008; 455:1061-1068. [PubMed: 18772890]

30. Verhaak RG, et al. Integrated genomic analysis identifies clinically relevant subtypes of glioblastoma characterized by abnormalities in PDGFRA, IDH1, EGFR, and NF1. Cancer Cell. 2010; 17:98-110. [PubMed: 20129251]

31. Pedersen MW, Meltorn M, Damstrup L, Poulsen HS. The type III epidermal growth factor receptor mutation. Biological significance and potential target for anti-cancer therapy. Ann Oncol. 2001; 12:745-760. [PubMed: 11484948]

32. Mellinghoff IK, et al. Molecular determinants of the response of glioblastomas to EGFR kinase inhibitors. N Engl J Med. 2005; 353:2012-2024. [PubMed: 16282176]

33. Galban CJ, et al. The parametric response map is an imaging biomarker for early cancer treatment outcome. Nat Med. 2009; 15:572-576. [PubMed: 19377487]

34. Galban CJ, et al. Prospective analysis of parametric MRI biomarkers: Identification of early and distinct glioma response patterns not predicted by standard radiographic assessment. Clin Cancer Res. 2011

35. Tsien C, et al. Parametric response map as an imaging biomarker to distinguish progression from pseudoprogression in high-grade glioma. J Clin Oncol. 2010; 28:2293-2299. [PubMed: 20368564]

36. Elkhaled A, et al. Magnetic Resonance of 2-Hydroxyglutarate in IDH1-Mutated Low-Grade Gliomas. Sci Transl Med. 2012; 4:116ra5.

37. Wei LH, et al. Changes in tumor metabolism as readout for Mammalian target of rapamycin kinase inhibition by rapamycin in glioblastoma. Clin Cancer Res. 2008; 14:3416-3426. [PubMed: 18519772]

38. Yoon TJ, Lee H, Shao H, Weissleder R. Highly magnetic core-shell nanoparticles with a unique magnetization mechanism. Angew Chem Int Ed Engl. 2011; 50:4663-4666. [PubMed: 21495138] 

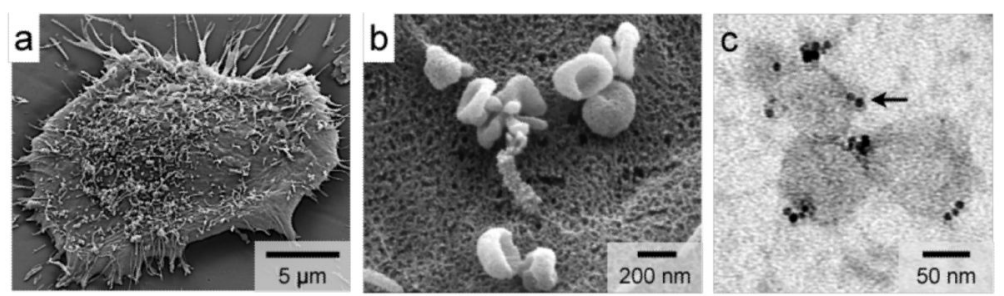

d
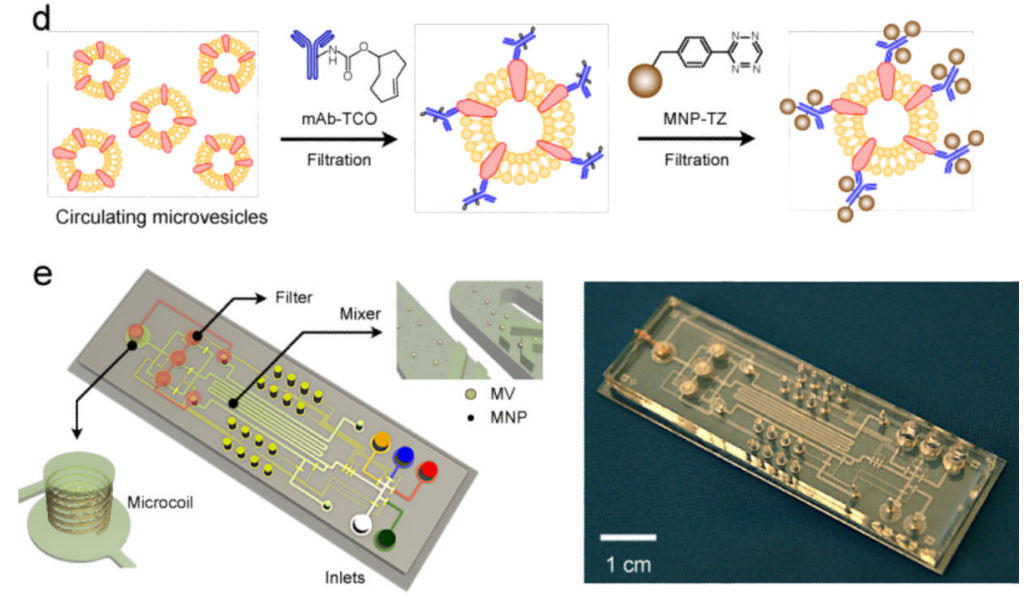

Figure 1. Human glioblastoma cells produce abundant microvesicles (MVs) which can be analyzed by micro nuclear magnetic resonance ( $\mu$ NMR)

(a) Scanning electron microscopy image of a primary human glioblastoma cell (GBM20/3) grown in culture, releasing abundant MVs. (b) High magnification image shows that many of the MVs on the cell surface assumed typical saucer-shaped characteristics of exosomes.

(c) Transmission electron microscopy image of MVs $(\sim 80 \mathrm{~nm})$ targeted with magnetic nanoparticles (MNPs) via CD63 antibody. The samples were purified by membrane filtration to collect small MVs. The MNPs appear as black dots (indicated by an arrow). (d) Labeling procedure for extravesicular markers. The two-step BOND-2 assay configuration uses bioorthogonal amplification chemistry to maximize MNP binding onto target proteins on MVs (not drawn to scale). (e) Microfluidic system for on-chip detection of circulating MVs. The system was designed to i) allow MNP-targeting of MVs, ii) concentrate MNPtagged MVs while removing unbound MNPs, and iii) provide in-line $\mu$ NMR detection. 
a

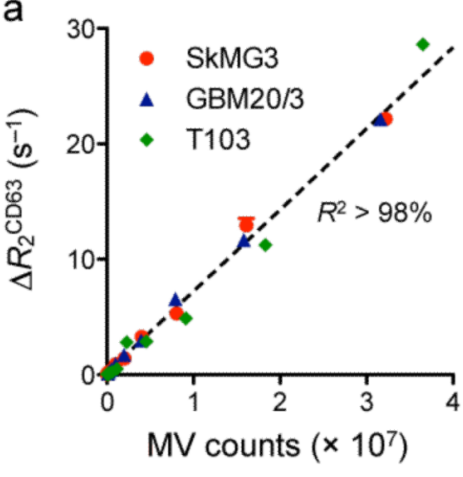

C

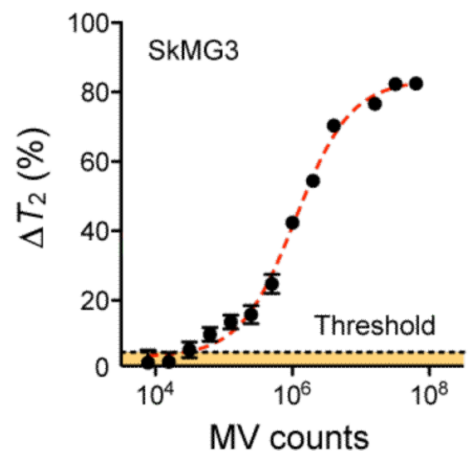

b

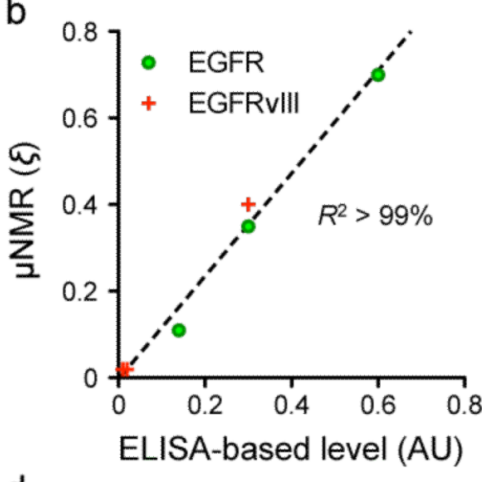

d

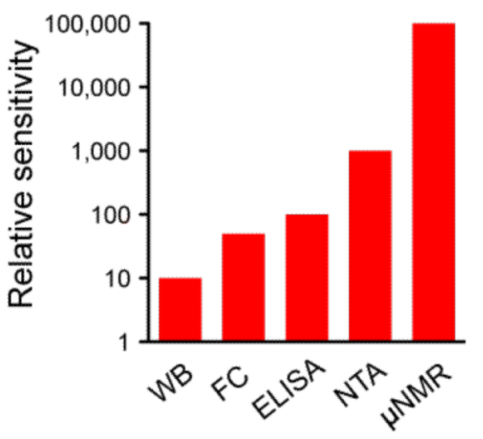

Figure 2. $\mu$ NMR assay for MV detection

(a) Correlation between $\mu$ NMR measurements for CD63 and MV numbers in a dilution series. MV numbers were estimated by nanoparticle tracking analysis (NTA). The transverse relaxation $\left(R_{2}\right)$, as determined by $\mu$ NMR, varied linearly with MV numbers $\left(R^{2}>98 \%\right)$. Importantly, the $R_{2}$ changes ( $\triangle R_{2}{ }^{\mathrm{CD} 63}$ ) were statistically identical across different cell lines $(P>0.16)$, which validates its use as a universal measure for quantitating MVs. Western blotting (Supplementary Fig. 4a) also revealed a consistent and higher expression of CD63 in the prepared MVs. (b) Using MVs from model cell lines, the expression levels of EGFR and EGFRvIII were measured by $\mu$ NMR. The MV expression $(\xi)$ of a target protein marker was obtained by normalizing a marker-associated $R_{2}$ against $R_{2}{ }^{\mathrm{CD}} 63 . \mu \mathrm{NMR}$ measurements showed excellent agreement $\left(R^{2}>99 \%\right)$ with fluorescence ELISA. (c) Detection threshold of $\mu$ NMR assay for MVs. With CD63-tagged MVs, the detection threshold of $\mu$ NMR, as measured by the relative changes in the transverse relaxation time $\left(T_{2}=1 / R_{2}\right)$ with respect to controls, was approximately $\sim 10^{4} \mathrm{MVs}$. (d) Comparison of MV detection sensitivity. In a series of MV dilution assays, $\mu$ NMR was considerably more sensitive than Western blotting (WB; Supplementary Fig. 4b), flow cytometry (FC), ELISA and NTA. All measurements were performed in triplicate, and the data is displayed as mean \pm s.e.m. 

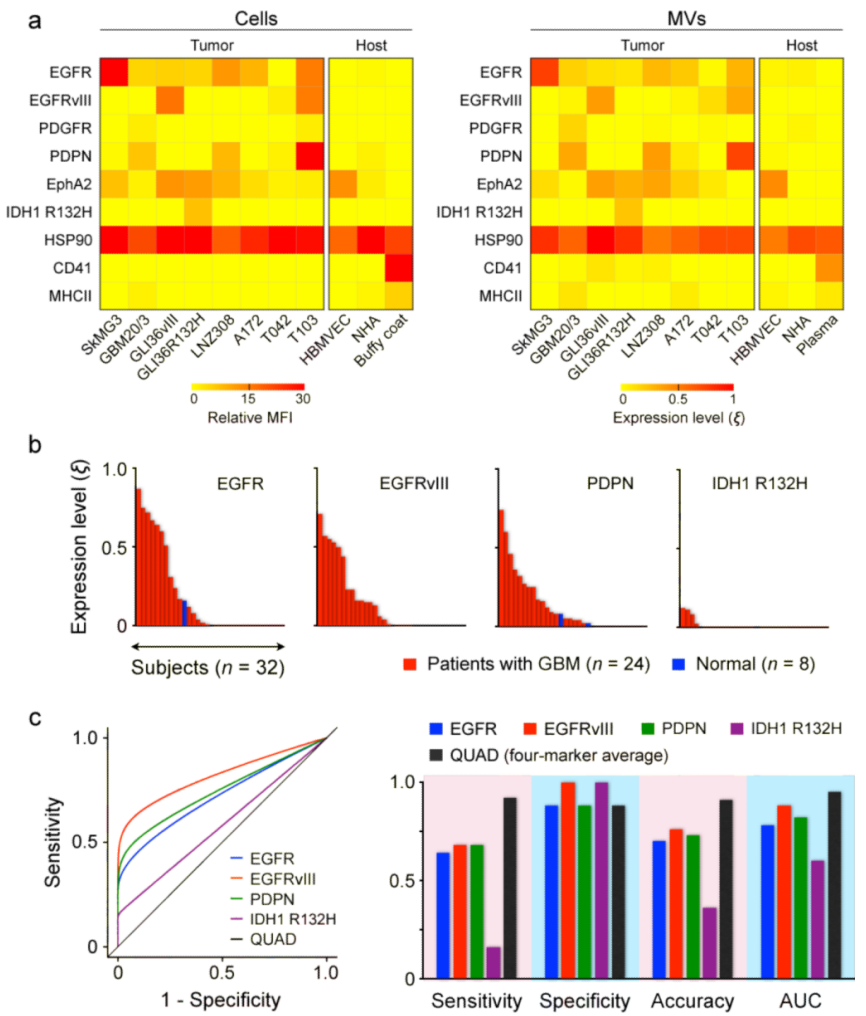

Figure 3. Protein typing of glioblastoma multiforme (GBM)-derived MVs from cell lines and patient samples

(a) GBM markers (EGFR, EGFRvIII, PDGFR, PDPN, EphA2 and IDH1 R132H), positive MV control marker (HSP90) as well as host cell markers (CD41, MHCII) were profiled in both parental cells (left) and their corresponding MVs (right). A four GBM marker combination (EGFR, EGFRvIII, PDPN and IDH1 R132H) was able to distinguish GBMderived MVs from host cell-derived MVs. HBMVEC, human brain microvascular endothelial cell; NHA, normal human astrocyte; buffy coat and plasma were isolated from whole blood donated by healthy volunteers. (b) Analysis of clinical patient samples. Waterfall plots show the expression levels of different biomarkers sorted from high (left) to low (right). Note the increased expression of EGFR and PDPN, as well as the unique expression of EGFRvIII and IDH1 R132H, in patient samples. (c) Receiver operating characteristic curves (left) were generated to compare the detection sensitivity, specificity and accuracy of each marker. Overall, the accuracy was $<76 \%$ for a single marker alone (right). When all markers were combined (QUAD), the detection accuracy considerably improved (> 90\%). AUC: area under curve. 

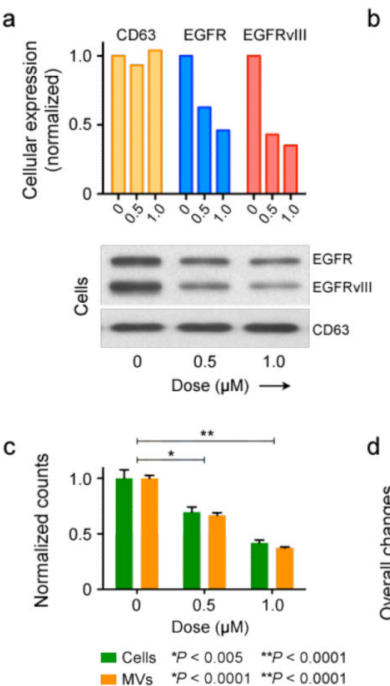

e

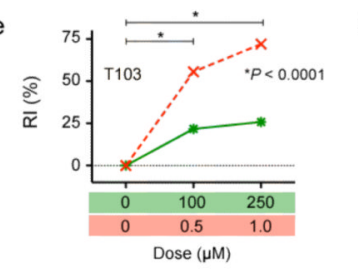

C* TMZ - - - Geldanamycin
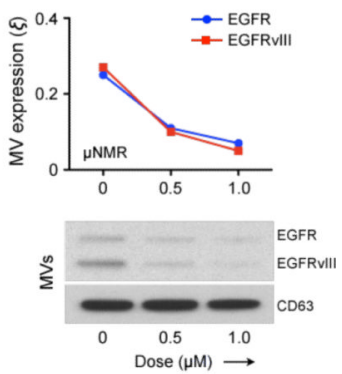

d
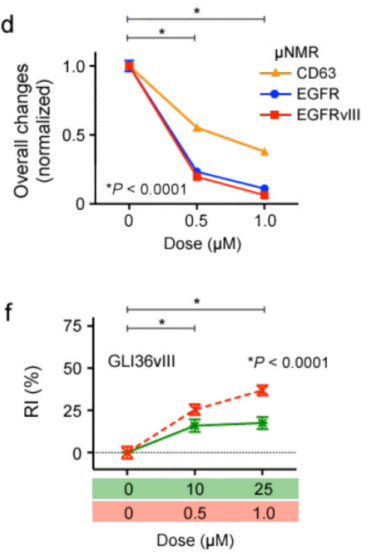

Dose $(\mu \mathrm{M})$

Figure 4. Effects of geldanamycin treatment on T103 GBM model

(a) On the cellular level, geldanamycin treatment did not alter the expression of CD63, but considerably reduced the amount of EGFR and EGFRvIII, as determined by flow cytometry and Western blotting. (b) Upon geldanamycin treatment, $\mu$ NMR detection showed that MVs exhibit a similar decrease in EGFR and EGFRvIII profiles ( $\xi$; normalized with respect to CD63 expression) as that observed in whole cells. (c, d) Total number of cells and MVs (c) decreased in a dose-dependent manner, upon drug treatment. However, the total EGFR and EGFRvIII levels in MVs (d) showed a steeper decline due to the combined effects of reduced MV number and decreased marker (EGFR, EGFRvIII) expression per MV. (e, f) To use MV readouts as an indicator for drug efficacy, a response index (RI) was defined, that recapitulates changes in both MV number and MV molecular expression. Compared to TMZ, the RI of geldanamycin was higher for both T103 (e) and GLI36vIII (f) cell lines due to the drug's ability to reduce both MV number as well as receptor expression. All changes with respect to untreated samples were statistically significant $(P<0.001)$. All analytical measurements were performed in triplicate, and the data is shown as mean \pm s.e.m. 


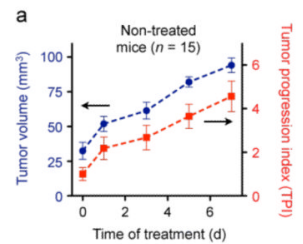

d

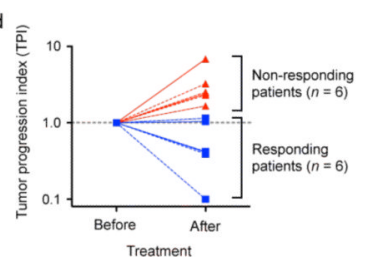

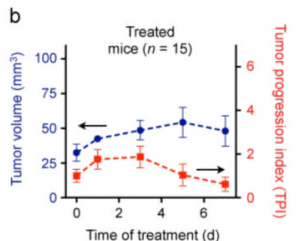
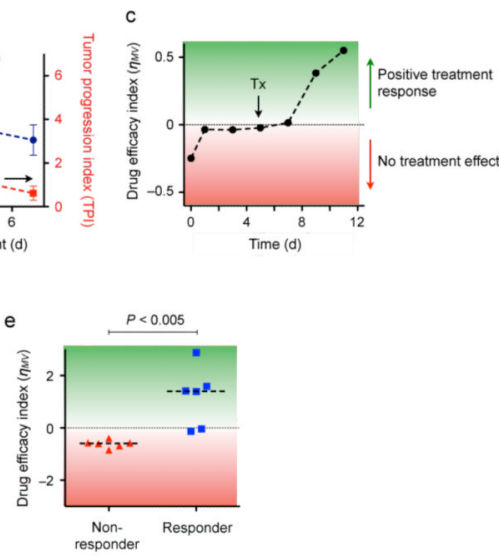

Figure 5. Analysis of circulating MVs in GBM mice and human patients undergoing treatment (a) Circulating MVs in untreated tumor-bearing animals $(n=15)$. The tumor progression index (TPI) is used to reflect changes in both MV number and MV molecular expression. Note the close correlation between increasing tumor volumes and TPI values over time. (b) In TMZ treated mice $(n=15)$, TPI values from $\mu$ NMR measurements revealed response to treatment before apparent changes in tumor size. (c) Since the decline rate of TPI represents a time-sensitive indicator of treatment efficacy, we define the drug efficacy index $\left(\eta_{M V}\right)$ as the temporal change in TPI ${ }^{-1}$. With TMZ treatment, $\eta_{M V}$ switched from negative (tumor progression) to positive (treatment response). (d, e) Clinical trial. Blood samples were collected from the same patients before and after TMZ/radiation treatment, and circulating MVs were profiled using $\mu$ NMR. Both TPI (d) and $\eta_{M V}(\mathbf{e})$ confirmed that longitudinal MV profiling can be used to predict treatment outcomes and differentiate between responders and non-responders. Dashed lines in (e) indicate the median values. 